\title{
Hybrid ZnO-organic semiconductor interfaces in photodetectors: A comparison of two near- infrared donor-acceptor copolymers
}

\author{
Alec Pickett ${ }^{1}$, Abhisek Mohapatra ${ }^{2}$, Amrit Laudari ${ }^{l}$, Soma Khanra ${ }^{1}$ Thulasi Ram ${ }^{2}$, Satish Patil ${ }^{2}$, and \\ Suchismita Guha ${ }^{1 *}$ \\ ${ }^{1}$ Department of Physics and Astronomy, University of Missouri, Columbia, MO 65211, USA \\ ${ }^{2}$ Solid State and Structural Chemistry Unit, Indian Institute of Science, Bangalore, India, 560012
}

\begin{abstract}
Hybrid organic-inorganic photodiode interfaces have gained significant interest due to their unique physical properties such as mechanical flexibility and high photosensitivity. Two diketopyrrolopyrrole (DPP)-based donor-acceptor copolymers with different backbone conformations are characterized in an inverted non-fullerene photodiode architecture using $\mathrm{ZnO}$ nano-patterned films as the electron transport layer. The DPP copolymer with a thienothiophene unit (PBDT-TTDPP) is more planar and rigid compared to the DPP system with a thiophene unit connecting the donor and acceptor moieties within the monomer (PBDT-TDPP). The hybrid interfaces were optimized by using poly(3-hexylthiophene) (P3HT) as the $p$-type layer for monitoring the critical thickness and morphology of the $\mathrm{ZnO}$ layer. The maximum photoresponsivity from a P3HT:ZnO photodiode was found to be $56 \mathrm{~mA} / \mathrm{W}$. The photoresponsivity of PBDT-TTDPP:ZnO photodiodes were found to be more than two orders of magnitude higher than PBDTTDPP:ZnO photodiodes, which is attributed to an enhanced transport of carriers due to the planar backbone conformation of the PBDT-TTDPP copolymer. Capacitance-voltage measurements from hybrid Schottky barrier interfaces further shed light into the nature of photocarriers and device parameters. Firstprinciples time-dependent density-functional theoretical calculations yield a higher absorptivity for the PBDT-TTDPP dimer compared to PBDT-TDPP.
\end{abstract}

KEYWORDS photodiodes; organic-inorganic interface; $\mathrm{ZnO}$; conjugated polymers; capacitance-voltage.

\footnotetext{
*Corresponding author E-mail address: guhas@missouri.edu
} 


\section{INTRODUCTION}

Hybrid inorganic/organic semiconducting interfaces have gained a lot of interest in solar cells, $[1,2]$ thin film transistors, $[3,4]$ and light-emitting diodes.[5, 6] The intrinsic $n$-type nature of several III-V and II-VI semiconductors and $p$-type nature of organic semiconductors offer a perfect platform for the realization of $p$ - $n$ junctions. For such junctions both $\mathrm{GaN}$ and $\mathrm{ZnO}$ interfaces have been utilized with $\pi$ conjugated polymers/molecules.[7, 8] Application of hybrid light-emitting diodes in microdisplays has been demonstrated by several groups.[9-11] $\mathrm{ZnO}$ is a non-toxic and environmentally friendly metal-oxide semiconductor, and is advantageous over other inorganic semiconductors since sol-gel chemistry allows the synthesis of high quality films with nano-patterning.[12-14] Nano-ridge patterning of $\mathrm{ZnO}$ in an inverted polymer solar cell was shown to improve the power conversion efficiency by almost $25 \%$ compared to planar $\mathrm{ZnO}$ nanoparticle based films.[12] Such a patterning strategy increases the interfacial area for electron collection. However, texturing of the $\mathrm{ZnO}$ layer results in current inhomogeneity, which is often mitigated by surface modification or adding a buffer layer between the $\mathrm{ZnO}$ film and the active layer.[13-16] Other strategies for increasing the interfacial area and the response of photodetectors include the usage of $\mathrm{ZnO}$ nanorods.[17-19]

The use of donor-acceptor conjugated polymers/oligomers has been on the rise in organic electronics due to the tunability of the bandgap energy and their structural property to support both electron and hole transport. Copolymers based on diketopyrrolopyrrole (DPP) core have attracted a lot of attention due to their high $p$-type as well as $n$-type carrier mobilities in organic field-effect transistors (FETs).[20-25] The right choice of side-chains exemplifies "order in disorder" in DPP systems, resulting in a highly interconnected network of the polymer chains, [26] enhancing transport properties in FETs. DPP copolymers blended with fullerenes have shown high power conversion efficiencies in solar cell structures.[27-29] Moreover, DPP based co- and statistical -polymers have served as a test-bench for probing charge transfer states, both experimentally[30, 31] and theoretically.[32]

Impedance spectroscopy provides a powerful tool to probe energy levels within the band gap of a material and has been widely used to characterize organic solar cells.[33-36] In particular, the junction 
region response of a pn diode has capacitive $(C)$ and conductive $(G)$ components, expressed in terms of the admittance $Y=G+j \omega C$, where $\omega$ is the angular frequency of the ac signal in radians/s. When reverse biased, the diode conductance is small and $Y \cong j \omega C$, and the pn junction diode is essentially equivalent to a capacitor. Capacitance-voltage $(\mathrm{C}-\mathrm{V})$ measurements are thus particularly useful to determine device parameters from Schottky barrier organic-inorganic planar heterojunction photodetectors.

In this work we use DPP-based copolymers with $\mathrm{ZnO}$ nanopatterned films in inverted photodetector structures without the use of any fullerene. The hybrid interfaces were optimized by using poly(3hexylthiophene) (P3HT) as the $p$-type layer for monitoring the thickness and morphology of the $\mathrm{ZnO}$ layer. The optimized thickness of the $\mathrm{ZnO}$ nanopatterned films lies between 300-500 nm. The two DPP copolymers with benzodithiophene (BDT) units have peak absorption in the $700 \mathrm{~nm}$ range and have distinct differences in their backbone conformation: PBDT-TTDPP, with a thienothiophene unit, has a planar backbone compared to the PBDT-TDPP copolymer with a thiophene unit connecting the donor and acceptor moieties. A higher charge transport mechanism in PBDT-TTDPP compared to PBDT-TDPP is seen in FET devices. Photodiodes with PBDT-TTDPP yield a photoresponsivity which is more than two orders of magnitude higher compared to PBDT-TDPP. Detailed capacitance-voltage and capacitancefrequency measurements from the photodiodes show subtle differences between the two DPP copolymers. These results are further compared with P3HT:ZnO and P3HT:PCBM based photodetectors. Density functional theory (DFT) and time-dependent density functional theory (TDDFT) calculations of the electronic energies and excitations from dimeric units of PBDT-TDPP and PBDT-TTDPP are also presented.

One may envisage cost-effective near infrared (IR) photodetectors without the use of fullerenes in the near future. Since DPP copolymers comprising donor-acceptor units provide a viable route towards a reasonable choice for near IR materials, it is equally important to understand the design rule of these materials. The polymer backbone and the linker unit between the donor and acceptor units play a major role in charge transfer, extraction, and thus the overall device performance. 


\section{EXPERIMENTAL METHODS}

\subsection{Materials}

All chemicals for the synthesis of the DPP copolymers were purchased from Sigma-Aldrich and were used without further purification. The solvents were analytical grade and were dried over sodiumbenzophenone and distilled before use. ITO-coated glass, on which $\mathrm{ZnO}$ and polymer films were deposited onto, was purchased from Ossila, as well as glass encapsulation slides and encapsulation epoxy. Zinc acetate dihydrate $\left(\mathrm{C}_{4} \mathrm{H}_{6} \mathrm{O}_{4} \mathrm{Zn} \cdot 2 \mathrm{H} 2 \mathrm{O}\right.$, ACS Reagent $\left.\geq 98 \%\right)$, ethanolamine $\left(\mathrm{C}_{2} \mathrm{H}_{7} \mathrm{NO}\right.$, ACS Reagent $\geq 99.0 \%$, and 2-methoxyethanol $\left(\mathrm{C}_{3} \mathrm{H}_{8} \mathrm{O}_{2}\right.$, anhydrous $\left.99.8 \%\right)$ used in fabricating the $\mathrm{ZnO}$ films were all acquired from Sigma-Aldrich. 1,2-dichlorobenzene (anhydrous 98\%) and chloroform (HPLC grade), the solvents used to create the polymer solutions, were acquired from Sigma-Aldrich and Fisher Scientific, respectively. P3HT (Poly(3-hexylthiophene-2,5-diyl)) was obtained from Rieke Metals, Inc. The gold (Au wire, 99.99\% pure) used for electrode evaporation was obtained from Kurt J. Lesker Company.

\subsection{Synthesis of DPP copolymers}

The synthesis of monomers; (4,8-bis(5-(2-ethylhexyl)thiophen-2-yl)benzo[1,2-b:4,5-b']dithiophene-2,6diyl)bis(trimethylstannane) (M1), 3,6-bis(5-bromothiophen-2-yl)-2,5-bis(2-octyldodecyl)pyrrolo[3,4c]pyrrole-1,4(2H,5H)-dione (M2) and 3,6-bis(5-bromothieno[3,2-b]thiophen-2-yl)-2,5-bis(2octyldodecyl)pyrrolo[3,4-c]pyrrole-1,4(2H,5H)-dione (M3) were performed by previously reported procedure.[37] For the desired copolymers, PBDT-TDPP and PBDT-TTDPP, palladium catalyzed Stille cross coupling polymerization method was used as shown in Scheme 1. 


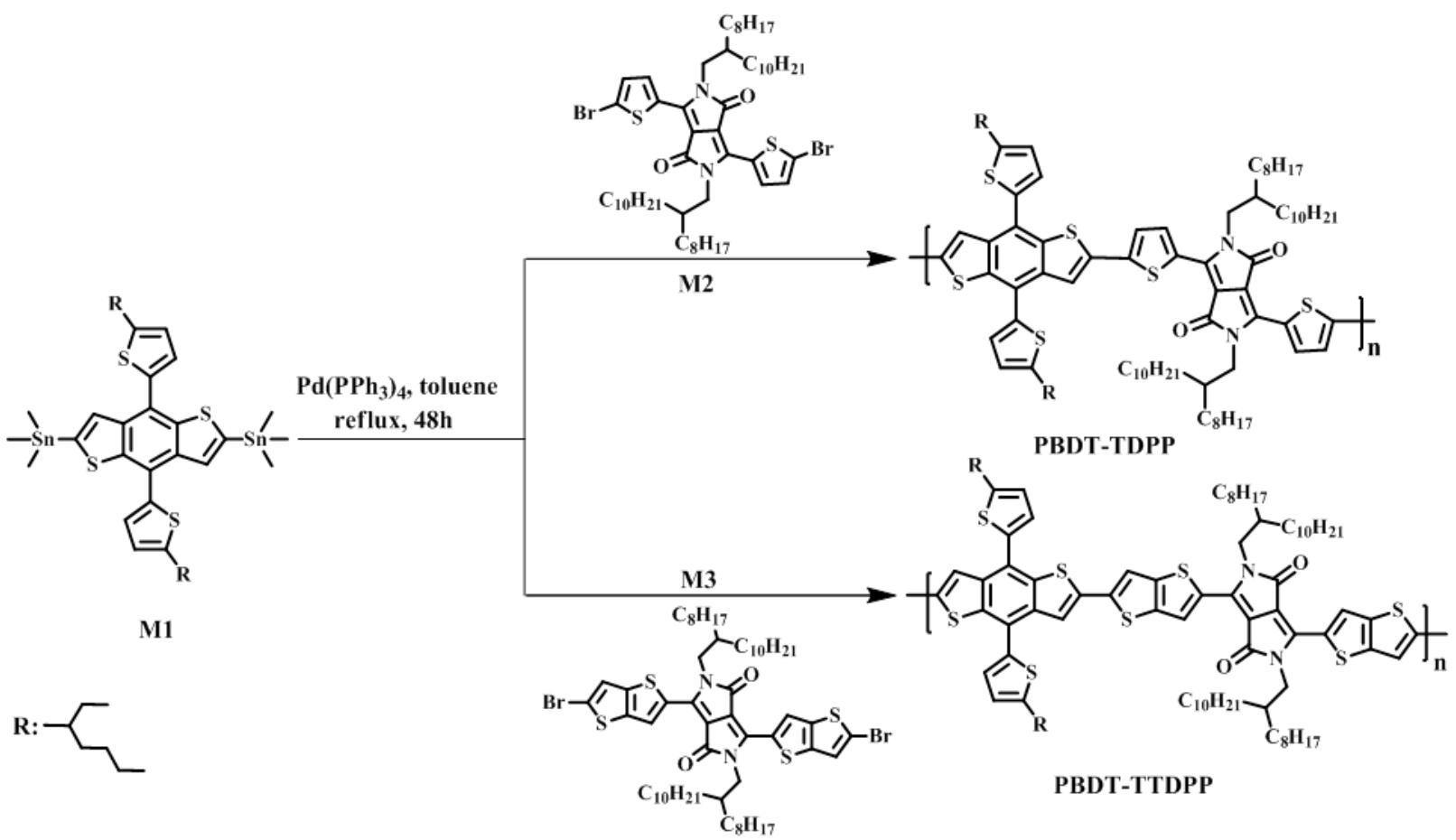

Scheme 1. Synthetic route to D-A copolymers

Monomers M1 $(0.10 \mathrm{mmol})$ and $\mathbf{M} 2$ or $\mathbf{M 3}(0.10 \mathrm{mmol})$ were dissolved in degassed toluene $(15 \mathrm{~mL})$ in a Schlenk tube. $\mathrm{Pd}\left(\mathrm{PPh}_{3}\right)_{4}(0.01 \mathrm{mmol})$ in dry toluene was added into monomer solution and purged under nitrogen for $15 \mathrm{~min}$. The reaction mixture was then refluxed for $48 \mathrm{~h}$ before cooling it down to room temperature and quenching with $2 \mathrm{M} \mathrm{HCl}$. Further, to the reaction mixture, EDTA solution was added followed by extraction with dichloromethane and dried over $\mathrm{Na}_{2} \mathrm{SO}_{4}$. The crude polymer was precipitated from the concentrated organic layer by addition of methanol. The precipitate was collected by filtration and then purified by Soxhlet extraction using methanol and acetone to remove residual starting materials or oligomers. Finally, the dark greenish solid was dried under vacuum to get pure PBDT-TDPP or PBDT-TTDPP. The molecular weight of the polymers was determined by gel permeation chromatography (GPC). For PBDT-TDPP: $\mathrm{Mw}=22.3 \mathrm{kDa}, \mathrm{Mn}=12.3 \mathrm{kDa}, \mathrm{PDI}=1.81$, and for PBDTTTDPP: $\mathrm{Mw}=229.7 \mathrm{kDa}, \mathrm{Mn}=86.8 \mathrm{kDa}$ PDI $=2.64$.

\subsection{Synthesis of nanopatterned $\mathrm{ZnO}$ films}

The $\mathrm{ZnO}$ nanopatterned films were fabricated using a sol-gel method. The solution was prepared by dissolving $1 \mathrm{~g}$ of zinc acetate dehydrate $\left(\mathrm{C}_{4} \mathrm{H}_{6} \mathrm{O}_{4} \mathrm{Zn} \cdot 2 \mathrm{H}_{2} \mathrm{O}\right)$ and $277 \mu \mathrm{L}$ ethanolamine $\left(\mathrm{C}_{2} \mathrm{H}_{7} \mathrm{NO}\right)$ in $10 \mathrm{~mL}$ 
of 2-methoxyethanol $\left(\mathrm{C}_{3} \mathrm{H}_{8} \mathrm{O}_{2}\right)$, then let to stir for at least 12 hours. The ITO coated glass substrates on which the $\mathrm{ZnO}$ film was deposited were treated with $\mathrm{NaOH} .10 \mathrm{~g}$ of sodium hydroxide $(\mathrm{NaOH})$ was dissolved in $100 \mathrm{~mL}$ of DI water and the substrates were left in the solution for 2 minutes. Immediately afterwards the substrates were sonicated in DI water and isopropanol for 10 minutes each. Sonication in DI water and isopropanol was done at least twice for each substrate followed by organic cleaning. Once the substrates were cleaned, the $\mathrm{ZnO}$ solution was spin-coated at $2000 \mathrm{rpm}$ for 60 seconds. While spinning, about $0.5 \mathrm{~mL}$ of the $\mathrm{ZnO}$ solution was dropped on each substrate via a pipette. The substrates were then transferred to a hot plate at room temperature and the temperature was increased at a rate of 50 ${ }^{\circ} \mathrm{C} /$ min until $275^{\circ} \mathrm{C}$, at which the film was left to anneal for 45 minutes. This spin-coating procedure was repeated multiple times to attain desired thickness and RMS roughness of the $\mathrm{ZnO}$ films. At the end of each day and after each $\mathrm{ZnO}$ film was completed, the films were annealed at $225^{\circ} \mathrm{C}$ in an oven in an oxygen atmosphere for at least 12 hours to reduce the conductivity of the $\mathrm{ZnO}$ films. Once fully annealed, an Aqua Regia solution (3:1:3 $\mathrm{HCl}: \mathrm{HNO}_{3}: \mathrm{H}_{2} \mathrm{O}$ diluted in $10 \mathrm{~mL}$ DI water) was used to expose the ITO contacts, then rinsed with isopropanol and DI water and dried with compressed air. The above procedure was done in ambient atmosphere. For some samples, the $\mathrm{ZnO}$ films were briefly treated with UV-ozone using a Harrick Plasma Cleaner at a chamber pressure of $250 \mathrm{mTorr}$ of $\mathrm{O}_{2}$ gas. The UV-ozone treatment was conducted for $20 \mathrm{~s}$.

\subsection{Hybrid photodetector fabrication}

The finished $\mathrm{ZnO}$ films were transferred to a nitrogen-filled glove box for polymer spin-coating and electrode deposition. All polymer solutions were prepared by dissolving each polymer in a 1:1 mixture by volume of 1,2-dicholorbenzene and chloroform. The P3HT solution, at a concentration of $20 \mathrm{mg} / \mathrm{mL}$, was

filtered using a $0.22 \mu \mathrm{m}$ polytetrafluorethylene (PTFE) membrane filter and spin-coated at $680 \mathrm{rpm}$ for 40 seconds then annealed at $115^{\circ} \mathrm{C}$ in an oven for 5 minutes. The PBDT-TDPP and PBDT-TTDPP solutions with a concentration of $15 \mathrm{mg} / \mathrm{mL}$ were used. The copolymers were spin-coated at $680 \mathrm{rpm}$ for 40 seconds and annealed at $130{ }^{\circ} \mathrm{C}$ for 20 minutes. After annealing the polymer layer, a $50 \mathrm{~nm}$ Au layer was 
thermally evaporated at a base pressure of $10^{-5}$ mbar. Finally, the active areas of all substrates were encapsulated under a glass slide with the Ossila epoxy, curing for 5 minutes under a UV lamp.

\subsection{Electrical and other Characterization}

Morphology of the $\mathrm{ZnO}$ films was determined using a Nanoscope IIIa Atomic Force Microscope (AFM) in tapping mode from Bruker Corporation. Multiple images were taken of 16x16 $\mu \mathrm{m}$ sample areas, which yielded the film thickness and roughness. Current-voltage measurements were conducted using a Keithley 2400 source meter and a Newport Oriel Apex Illuminator solar simulator under AM 1.5 conditions. Responsivity was measured using a Keithley 2400 source meter, Stanford Research Systems SR810 DSP lock-in amplifier, and a CM110 1/8 m monochromator from Spectral Products. To estimate the responsivity, the photocurrent measured from the device under study was divided by the incident lamp power, which was calibrated using a Si photodetector. All photoresponsivity spectra shown in this work are at zero bias condition. Capacitance measurements were taken using a Hewlett Packard 4284A LCR Meter. For all electrical measurements, the diodes were loaded into an Ossila switchboard.

\section{RESULTS AND DISCUSSIONS}

\subsection{Nanopatterned $\mathrm{ZnO}$ films with varying thickness in P3HT photodetectors}

The $\mathrm{ZnO}$ films were characterized by Raman scattering (Figure S1) and AFM. The thickness of the nanopatterned $\mathrm{ZnO}$ layer on ITO was varied to find out the optimum architecture in planar heterojunction devices. Due to the patterned profile of the film, there is some variation of the thickness across the film. Figure 1 (b) shows the AFM images of three $\mathrm{ZnO}$ films with different thicknesses. The nanopatterning in the films forms ridge-like structures. Films that are less than $90 \mathrm{~nm}$ have large micron shaped features; between $300-500 \mathrm{~nm}$ thickness, the $\mathrm{ZnO}$ films reach their optimum features with isotropic wrinkle pattern with a characteristic fractal length.[14] Beyond $700 \mathrm{~nm}$, the wrinkles are seen to grow in size. Photodetectors were fabricated by depositing identical layers of P3HT (of thickness $\sim 100 \mathrm{~nm}$ ) on the $\mathrm{ZnO}$ films. The responsivity (Figure 1 (c)) is seen to be the highest for the 9 layered $\mathrm{ZnO}(\sim 300 \mathrm{~nm})$

device, beyond which the responsivity is seen to decrease. It also shows the best I-V characteristics 
(Figure 1 (d)). The 9 layered $\mathrm{ZnO}$ film was found to have the largest RMS roughness (Table 1), which corresponds to the amount of nanopatterning of the film. One inherent problem with $\mathrm{ZnO}$ is its conductivity. Although the $\mathrm{ZnO}$ films were annealed in an oxygen atmosphere for several hours in order to make them insulating, the diodes do show a small current upon reverse biasing (under dark conditions).
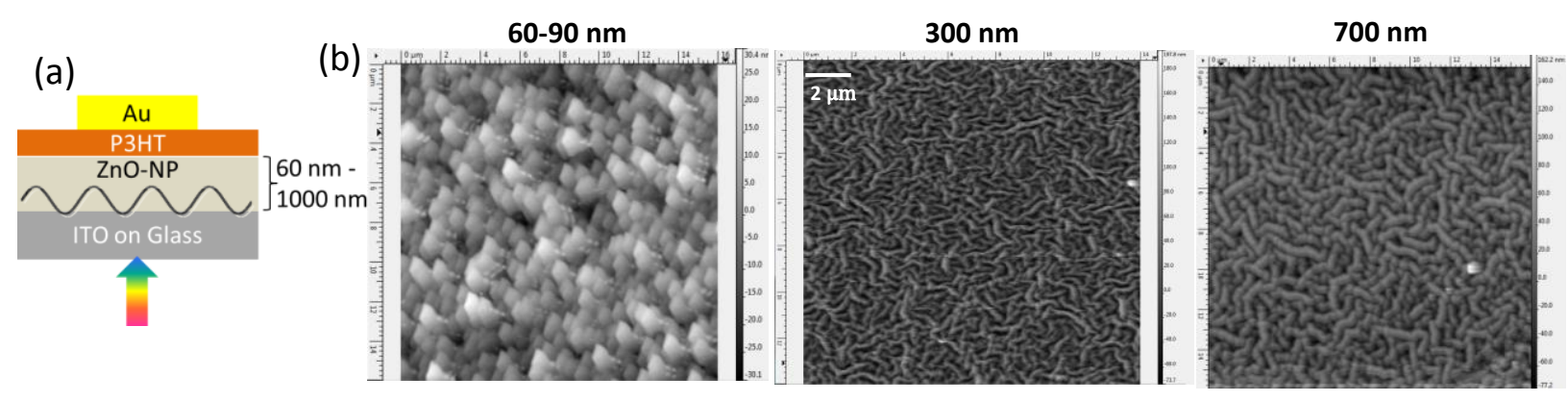

(c)
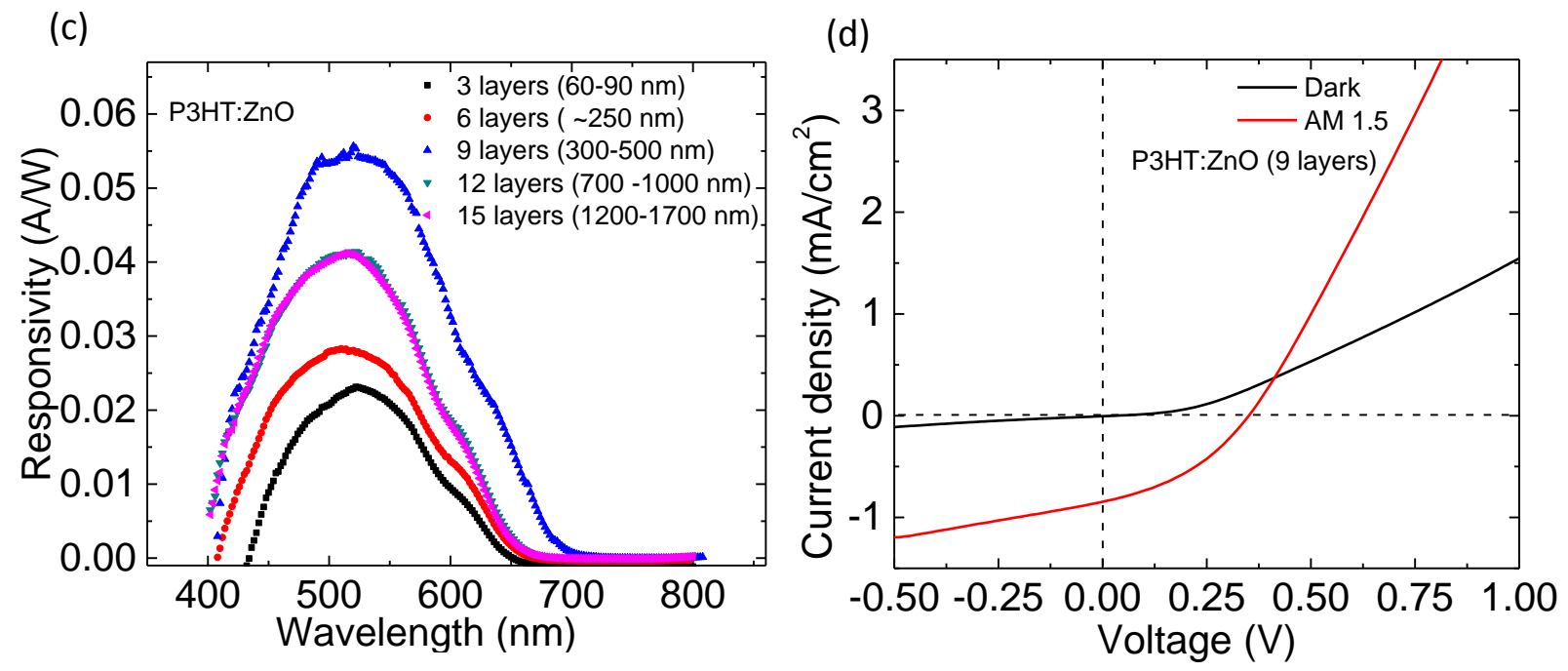

Figure 1. (a) Diode architecture and illumination side for all electrical measurements; (b) AFM images of 3, 9, and 12 layered $\mathrm{ZnO}$ films, respectively; (c) Photoresponsivity curves of P3HT on ZnO nanopatterned films of varying thickness; (d) Current-voltage curves of the 9 layered $\mathrm{ZnO}$ film with P3HT under dark and AM 1.5 conditions.

Table 1. ZnO film characteristics followed by the peak value of P3HT:ZnO responsivities. Values given are averages over six similar devices while values in parentheses are the maximum values found.

\begin{tabular}{cccc}
\hline Number of layers & Thickness $(\mathrm{nm})$ & RMS roughness $(\mathrm{nm})$ & Responsivity $(\mathrm{mA} / \mathrm{W})$ \\
\hline 3 & $60-90$ & $8.95 \pm 1.23$ & $14.7 \pm 4.8(23.1)$ \\
6 & $200-300$ & $18.40 \pm 1.50$ & $19.3 \pm 6.3(28.3)$ \\
9 & $300-500$ & $29.08 \pm 2.12$ & $46.1 \pm 10.6(55.6)$ \\
12 & $700-1000$ & $21.65 \pm 3.02$ & $35.7 \pm 4.0(41.4)$ \\
15 & $1200-1700$ & $22.97 \pm 2.44$ & $34.9 \pm 5.5(41.1)$ \\
\hline
\end{tabular}


The efficacy of hybrid photodetectors was also tested with $\mathrm{ZnO}$ films grown using pulsed laser deposition (PLD) and further compared with P3HT layers using a conventional architecture (ITO/PEDOT-PSS/P3HT/Ca-Al) but without any fullerene. The ZnO PLD films of thickness $\sim 40 \mathrm{~nm}$ were grown on ITO coated glass similar to Ref. [38] and annealed in air at $200{ }^{\circ} \mathrm{C}$ for a few hours before depositing the P3HT layer. From Figure 2, it is seen that PLD grown smooth ZnO films result in a maximum responsivity which is almost an order of magnitude smaller than the optimal nanopatterned $\mathrm{ZnO}$ film. Since the external quantum efficiency $(E Q E)$ of the photodiode is related to its responsivity $(R)$ by: $E Q E(\lambda)=R(\lambda) E(\lambda)$, where $E(\lambda)$ is the incident photon energy, the maximum $E Q E=0.13$ for P3HT:ZnO (nanopatterned film) shown in Figure 2. The EQE value obtained here is higher compared to other planar P3HT:ZnO photodiodes reported in the literature without the use of fullerenes.[39] The dashed line represents the responsivity from a pristine P3HT film sandwiched between ITO and Ca-Al electrodes. We further fabricated a P3HT:PCBM device using a standard architecture: ITO/PEDOTPSS/P3HT:fullerene/Ca-A1, which shows a maximum responsivity of $240 \mathrm{~mA} / \mathrm{W}$ at $500 \mathrm{~nm}$ (see Figure S2).

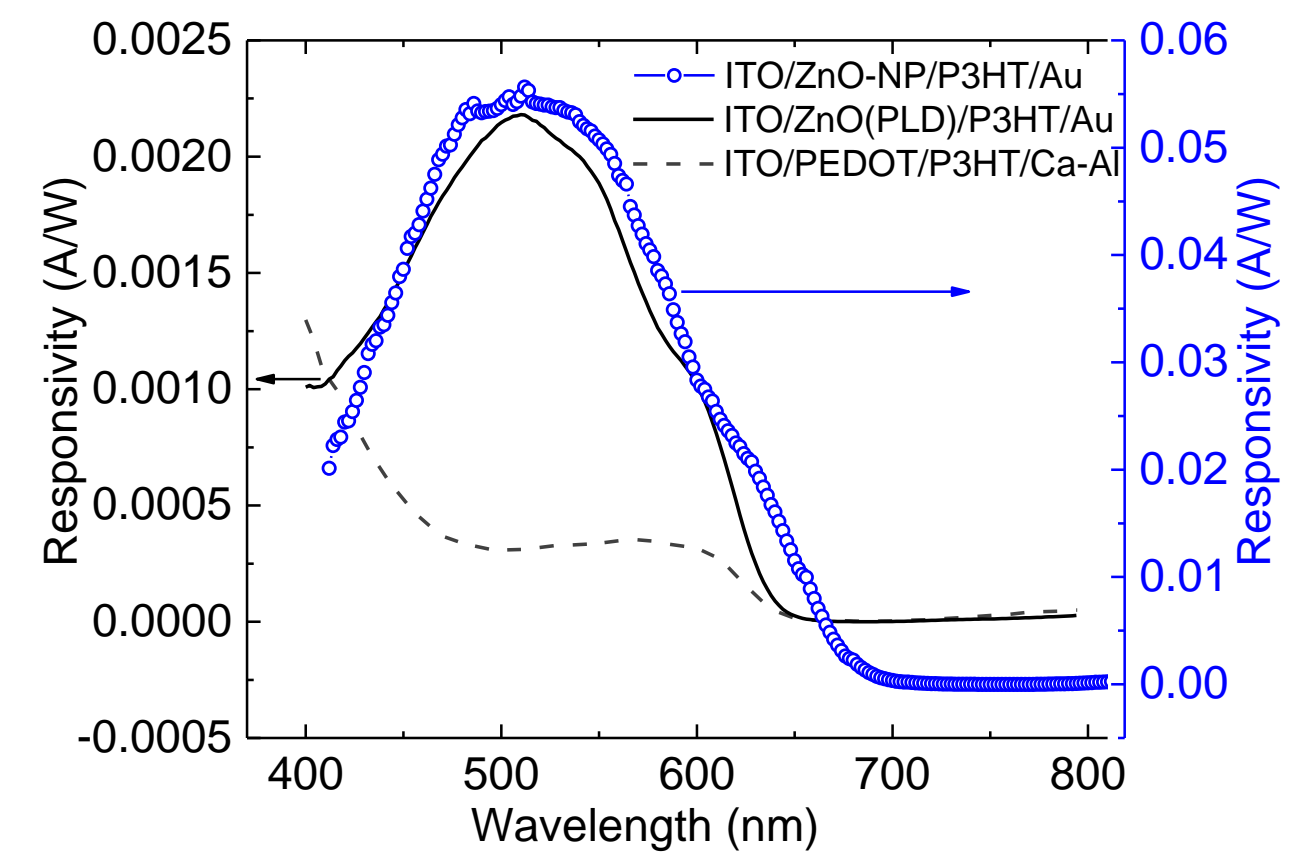

Figure 2. Responsivity of P3HT:ZnO devices using PLD grown and nanopatterned $\mathrm{ZnO}$ films. The dashed line is from a $\mathrm{P} 3 \mathrm{HT}$ device without any $\mathrm{ZnO}$ layer. 


\subsection{Photoresponsivity of DPP:ZnO photodiodes}

The optimized $\mathrm{ZnO}$ nanopatterned structures were used for fabricating photodetectors with the two DPP copolymers. Both copolymers exhibit ambipolar properties in FETs. The $n$-type nature is more pronounced in PBDT-TDPP, as seen clearly in metal-insulator-semiconductor (MIS) diodes. Capacitancevoltage $(\mathrm{C}-\mathrm{V})$ characteristics from PBDT-TDPP MIS diodes on $\mathrm{Al}_{2} \mathrm{O}_{3}$ dielectric show an accumulation region when the applied gate voltage is positive, thus demonstrating the accumulation of electrons at the semiconductor-dielectric interface (Figure S3). Such a behavior is not observed in PBDT- TTDPP MIS diodes.

Figure 3 compares the absorption and the photoresponsivity spectra of the two DPP copolymers. Similar to other donor-acceptor system, the absorption spectrum shows two prominent peaks. [31, 40] The high energy peak at approximately $350 \mathrm{~nm}$ is the $\pi-\pi^{*}$ transition, and the low energy broad peak centered at $700 \mathrm{~nm}$ is the intramolecular charge transfer (ICT) peak. It has been proposed that the formation of the ICT state stabilizes the donor-acceptor complexes, which are covalently bound along the polymer backbone.[41] Although the absorption spectra of the two DPP copolymers are similar, their photoresponsivities from hybrid photodiodes are quite different. The maximum responsivity of PBDTTTDPP:ZnO photodiodes is $2.2 \mathrm{~mA} / \mathrm{W}$ at $730 \mathrm{~nm}$, and the maximum responsivity of PBDT-TDPP:ZnO photodiodes is $0.012 \mathrm{~mA} / \mathrm{W}$ at $680 \mathrm{~nm}$. The specific detectivity, which is defined as $D^{*}=$ $R A^{1 / 2} /\left(2 q I_{\text {dark }}\right)^{1 / 2}$, where $R$ is the responsivity, $A$ is the area, $q$ is the electronic charge, and $I_{\text {dark }}$ is the dark current, is tabulated in Table S1 (Supporting Information) for the various photodetectors. Ozone treatment of the $\mathrm{ZnO}$ film, which fills oxygen vacancies at the surface, reducing the density of recombination sites, has shown significant improvement in ZnO-P3HT:PCBM devices.[42] We used a similar technique for a P3HT:ZnO and a PBDT-TTDPP:ZnO device; a slight improvement in the dark current and detectivity were observed. 
We further fabricated a PBDT-TTDPP:PCBM diode (using a standard solar cell architecture) and found the peak responsivity to be $160 \mathrm{~mA} / \mathrm{W}$. This translates to an $\mathrm{EQE}$ of $\sim 0.3$, which compares well with other DPP-based IR photodetectors where PCBM is used.[43] Since the goal of this work is to demonstrate the efficacy of the donor-acceptor based-DPP copolymers in non-fullerene based photodetectors, both responsivity and capacitance measurements are made from planar nanopatterned $\mathrm{ZnO}$ devices without PCBM.
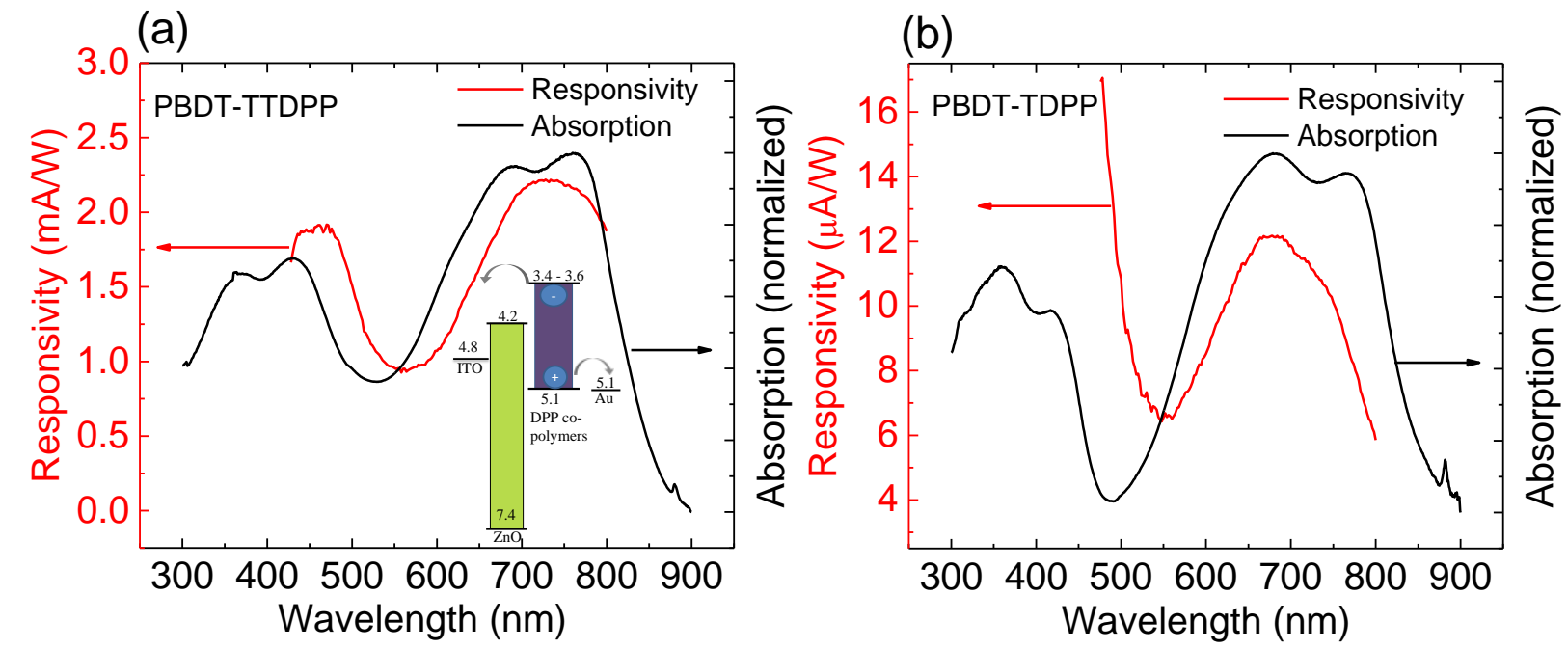

Figure 3. Absorption from pristine polymer films and responsivity from the photodiodes. (a) PBDTTTDPP and (b) PBDT-TDPP. The inset in (a) shows the energy levels in eV.

In order to understand the low photoresponse from PBDT-TDPP photodiodes, we consider several attributes of this copolymer. The copolymer is more $n$-type compared to PBDT-TTDPP as seen in C-V measurements from MIS structures (Figure S3), which for planar heterojunction photodiode architecture (with $n$-type $\mathrm{ZnO}$ ) may result in a bottleneck. However, it must be stated that both copolymers are predominantly $p$-type, as clearly seen in their FET transport (Figure S4). FETs were fabricated using both copolymers on $\mathrm{SiO}_{2}$ and $\mathrm{Al}_{2} \mathrm{O}_{3}$ gate dielectrics. The $p$-type carrier mobility $\left(\mu_{\mathrm{FET}}\right)$ of PBDT-TDPP is an order of magnitude lower than that of PBDT-TTDPP (Figure S4 (b) and (d)). The difference in charge transport properties may be attributed to the backbone conformation of the two copolymers. The thienothiophene linker between the donor-acceptor moieties in PBDT-TTDPP makes the copolymer more 
planar and rigid compared to the thiophene unit in PBDT-TDPP, enhancing transport properties. We note that the FET properties may be tuned by the choice of the dielectric layer: ALD grown $\mathrm{Al}_{2} \mathrm{O}_{3}$ dielectric layer lowers the operating voltage and enhances on/off ratios in PBDT-TTDPP FETs.

The linker unit between the donor-acceptor moieties plays a large role in the charge transfer process. Using a new visualization scheme, the particle-hole map (PHM),[44] for the spatial analysis of excitations in organic molecules, it was shown that DPP based donor-acceptor systems with thiophene versus phenyl linkage units show significant differences in their PHM; the phenyl linker is passive in the excitation process with a partial cancellation of charge transfer from the donor moiety to the DPP unit.[32] The theoretical results agreed well with the photoresponsivity of phenyl and thiophene based DPP solar cells.[30] The orders of magnitude lower photoresponsivity in PBDT-TDPP:ZnO photodiodes signals a passive charge transfer with the thiophene unit. The thienothiophene unit appears to play a coordinated role in the charge transfer process, resulting in high charge dissociation at the $\mathrm{ZnO}$ interface.

DPP-thienothiophene copolymers show a remarkably high absorption coefficient, which is attributed to a high persistence length of the polymer.[45] Moreover, the molecular weight of the polymer also scales with the extinction coefficient. We note that although both DPP copolymers used in this work have high molecular weights, PBDT-TTDPP is higher than PBDT-TDPP. The difference in molecular weights may contribute to a somewhat lower responsivity in PBDT-TDPP. However, more than two orders of magnitude difference in the responsivity and the differences in the $\mathrm{C}-\mathrm{V}$ behavior as seen in Section 3.3.2 cannot be explained solely on the molecular weight difference between the two copolymers. The backbone conformation and the thienothiophene linker unit in PBDT-TTDPP play a role in enhancing the transport of carriers.

Modulated photocurrent measurements from DPP photodiodes are shown in Figure 4 with a $785 \mathrm{~nm}$ excitation source $(30 \mathrm{~mW})$. These were measured under reverse bias conditions with the excitation source being on for $10 \mathrm{~s}$ and off for $5 \mathrm{~s}$. Both systems clearly show an on/off ratio of $10^{2}$ in the semi-log plot, although the magnitude of the photocurrent varies. The PBDT-TTDPP:ZnO photodiodes show a similar response as P3HT:ZnO photodiodes (when excited with a $532 \mathrm{~nm}$ source), where the output current 
closely follows the signal from the modulated light source. The photocurrent in PBDT-TDPP:ZnO decays more slowly.

The response time, which is another criterion for photodetectors, is defined in terms of the rise and fall times. The rise (fall) time is defined as the time interval from $10 \%(90 \%)$ to $90 \%(10 \%)$ of the photocurrent with respect to the dark current after the incident light is switched on (off). Using this criterion, we obtain for PBDT-TTDPP (PBDT-TDPP) an average rise time of $107 \mathrm{~ms}$ (368 ms) and fall time of $66 \mathrm{~ms}(575 \mathrm{~ms})$. The fast rise and fall times in PBDT-TTDPP:ZnO is comparable to detectors using vertical junction graphene oxide.[46]

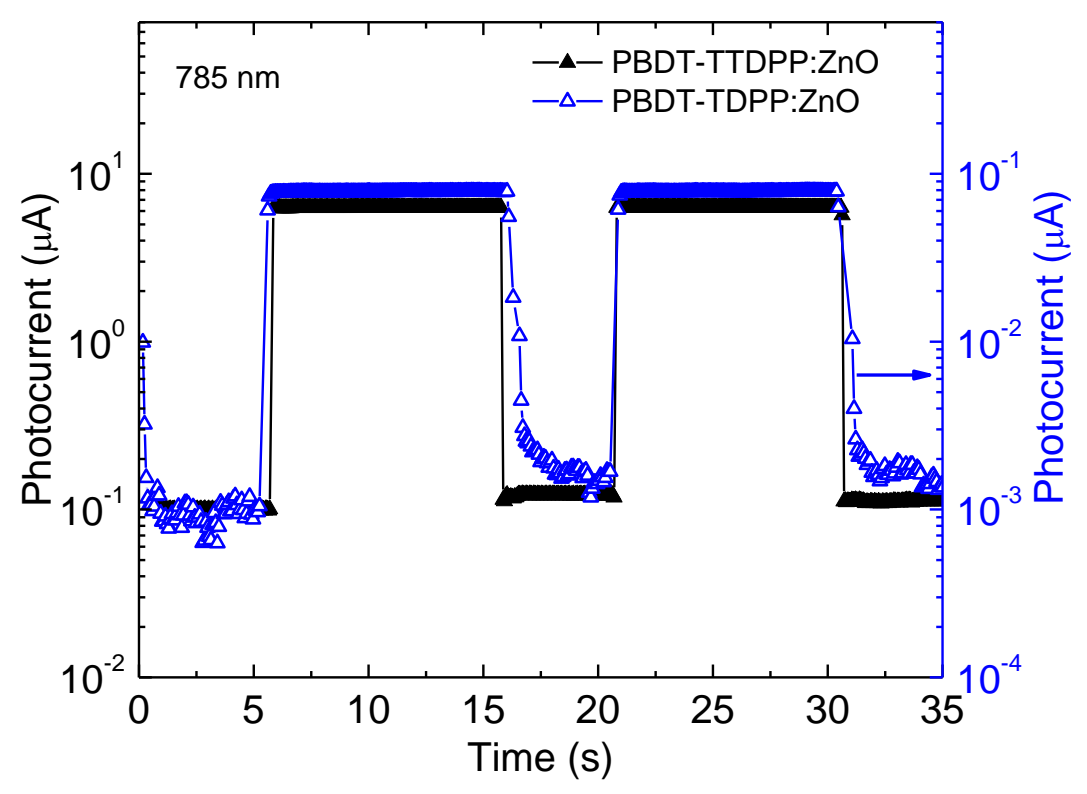

Figure 4. Photoresponse from DPP copolymer based photodiodes under periodic on-off cycles of $30 \mathrm{~mW}$ of $785 \mathrm{~nm}$ excitation.

In order to explain whether the differences in photoresponsivity from the two DPP copolymers originate from variations in the polymer itself or due to device parameters, we have carried out detailed $\mathrm{C}-\mathrm{V}$ (including capacitance-frequency) measurements from the Schottky diodes. These measurements are further compared with standard bulk heterojunction solar cells and planar P3HT:ZnO photodiodes.

\subsection{Capacitance measurements}


A small ac signal superimposed on the de reverse bias conditions of a pn junction diode results in a charge fluctuation, altering the depletion width about its equilibrium value. The charge fluctuation due to the ac response is similar to a parallel plate capacitor. One can further estimate the built-in voltage, $V_{B I}$, from $1 / C^{2}$ versus voltage data using the Mott-Schottky equation,[47]

$$
\frac{1}{C^{2}}=\frac{2}{A^{2} q \varepsilon N_{A}}\left(V_{B I}-V\right),
$$

where $A$ is the contact area, $q$ is the electron charge, $\varepsilon$ is the dielectric constant of the semiconductor, $N_{A}$ is the doping density, and $V$ is the applied bias. From a linear fit of the reverse-bias in a $\mathrm{C}^{-2}-\mathrm{V}$ plot, the builtin voltage can be found from the $x$-intercept. Consistent with eq. (1), for a metal-semiconductor diode the capacitance systematically decreases with an increased reverse bias.

In P3HT-PCBM based solar cell architectures, the work function difference between ITO and the metal anode results in a built-in field which induces a local electric polarization in the medium. The C-V measurements reveal a peak at a small bias voltage. $[48,49]$ The electrical polarization due to the interpenetrating donor-acceptor molecules builds surface charges near the electrodes, resulting in an effective charge which is further altered upon photoexcitation. As explained by Zhao et al.[48] the peak in a C-V sweep may be understood as follows: the effective capacitance increases as the applied voltage changes from $0 \mathrm{~V}$ to a small finite value (where the applied field negates the built-in field). Decreasing the effective field leads to an increase in effective charges (and thus an increased capacitance). Beyond the peak voltage, the opposing injected charges decrease the overall surface charge, and thus a decrease in capacitance is observed.

An ideal planar pn junction, however, shows a different $\mathrm{C}-\mathrm{V}$ characteristics compared to BHJs. A decreasing value of capacitance in the reverse bias region is an indication of an increase in the depletion width. With increasing positive voltages there is then an increase in the capacitance due to a decrease in the depletion width, and under forward bias conditions, the capacitance nearly remains a constant. A decrease in capacitance under forward bias conditions is typically associated with electrically active deep 
levels.[50] Next, we highlight the differences in $\mathrm{C}-\mathrm{V}$ behavior for bulk heterojunction and planar P3HT:ZnO junction diodes.

\subsubsection{P3HT:ZnO and P3HT:fullerene diodes}

There are several works that report impedance measurements from P3HT:PCBM and other bulk heterojunction $(\mathrm{BHJ})$ solar cells; both forward bias and reverse bias capacitance measurements have been used for determining defect densities.[51-53] The $\mathrm{C}-\mathrm{V}$ characteristics of a $\mathrm{P} 3 \mathrm{HT}: \mathrm{PC}_{70} \mathrm{BM}$ diode at different frequencies are shown in Figure 5 (a). The BHJ device shows an increased capacitance as the device is driven to the forward bias condition, and beyond $0.8 \mathrm{~V}$ the capacitance again decreases. The decrease in capacitance is attributed to a recombination of injected and surface charges (due to the local polarization). The devices were also illuminated by an excitation source at $532 \mathrm{~nm}$ (inset of Figure 5 (a)). The effective capacitance increases with the intensity of the incident light due to an enhanced generation of photocarriers. The peak capacitance is seen to shift to slightly lower voltages due to the injected carriers balancing the photoinduced carriers. The shift corresponds to the accumulation of photoinduced charges at the interface, lowering the effective potential barrier.

For an ideal $p n$ junction, the capacitance nearly remains a constant in the forward bias condition, as seen for one of our best performing P3HT:ZnO diodes (Figure 5 (b)). This device shows no hysteresis sweeping from positive to negative voltage and vice versa. Under $532 \mathrm{~nm}$ illumination, the capacitance is seen to increase, as seen in the inset of Figure 5 (b). 
(a)

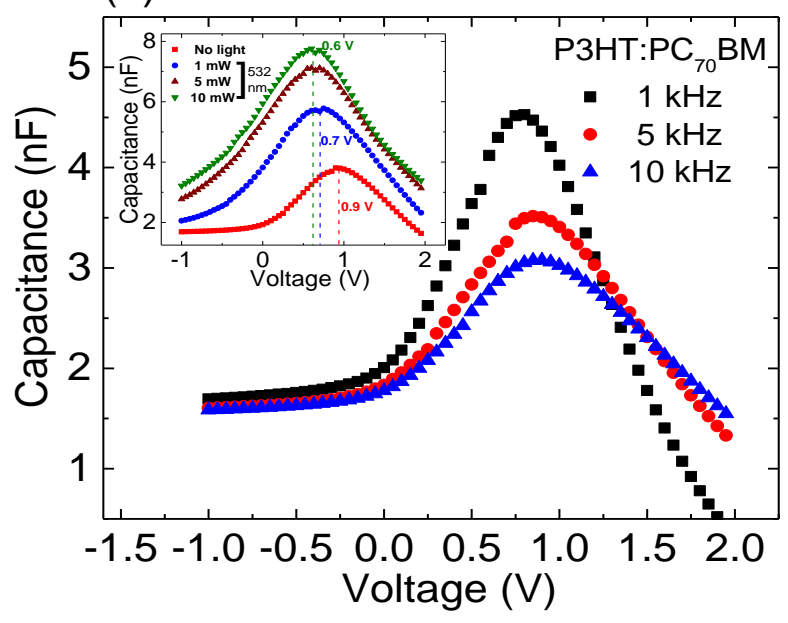

(b)

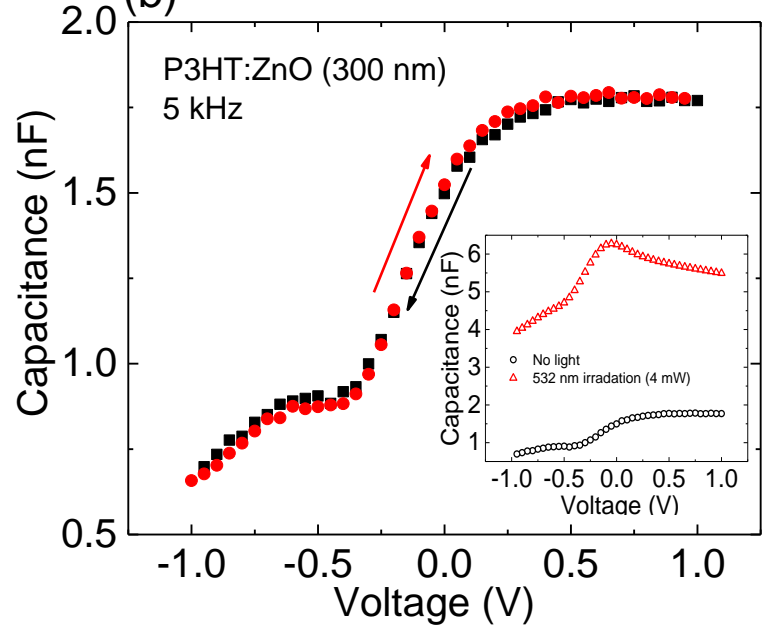

Figure 5. (a) Capacitance versus voltage from a $\mathrm{P} 3 \mathrm{HT}: \mathrm{PC}_{70} \mathrm{BM}$ diode at different frequencies. The inset shows the photoexcitation capacitance at $5 \mathrm{kHz}$. With increase in intensity of the incident excitation at $532 \mathrm{~nm}$, the capacitance increases and the peak shifts to lower voltages. (b) Capacitance versus voltage for a P3HT: $\mathrm{ZnO}$ diode. The $\mathrm{ZnO}$ thickness for this device was $\sim 300 \mathrm{~nm}$. The inset shows the capacitance under the illumination at $532 \mathrm{~nm}$.

Capacitance versus frequency (C-F) measurements are used to determine the density of deep trap states.[54] C-F plots for P3HT devices obtained under zero-bias conditions are shown in Figure 6. All three photodiodes show a similar performance with a drop in the capacitance beyond $100 \mathrm{kHz}$. The drop indicates a lack of the trapped charges in the active layer to respond to the ac frequency. Since the inverted and the regular solar cell show a similar C-F response, the trapped charges most likely relate to the P3HT layer. 


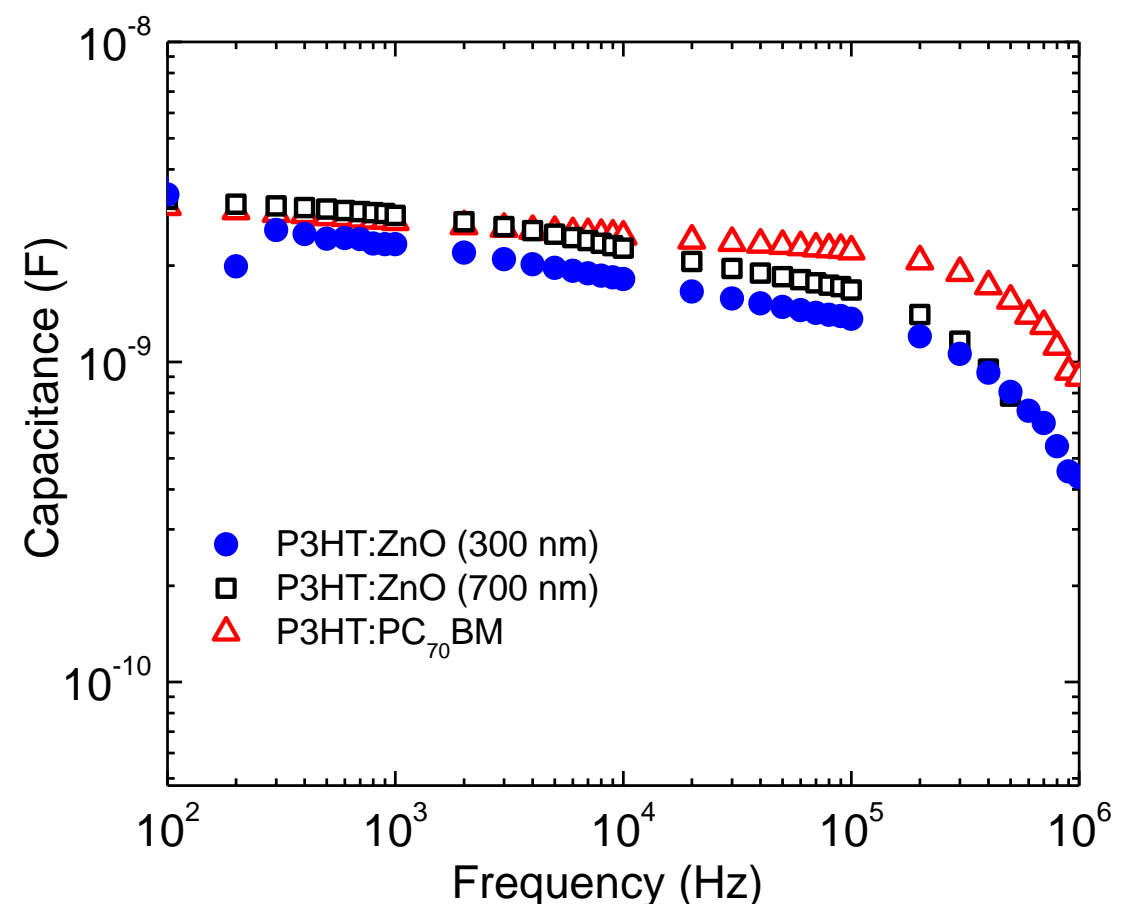

Figure 6. Capacitance versus frequency for the P3HT based diodes in a log-log plot.

\subsubsection{DPP: $\mathrm{ZnO}$ diodes}

$\mathrm{C}-\mathrm{V}$ measurements (at $5 \mathrm{kHz}$ ) for the DPP:ZnO photodiodes are shown in Figure 7. Both DPP:ZnO photodiodes show a sharp increase in capacitance as the voltage is swept more positive in the reverse bias region, followed by a sharp decrease in the forward bias region. The decrease in capacitance in the forward bias region could be a combination of two effects: recombination of surface charges by injected charges and a trapping in the deep levels. Curiously, the $\mathrm{C}-\mathrm{V}$ data under illumination is very different for the two samples as seen in Figure 7 (a) and (b). In both cases the illumination was with a 785 nm diode laser, where the absorption in the copolymers is strong. The PBDT-TDPP:ZnO photodiodes do not show any change in $\mathrm{CV}$ with illumination. PBDT-TTDPP:ZnO, however, displays a change in $\mathrm{CV}$ with illumination. The increase of the capacitance is again due to the generation of photocarriers and the central peak position shifts to higher voltages. Upon photoexcitation in PBDT-TTDPP:ZnO, there are two peaks that emerge in the $\mathrm{C}-\mathrm{V}$ characteristics, a shoulder centered at $-0.35 \mathrm{~V}$ and a peak at $0.15 \mathrm{~V}$. C-V 
peaks in the forward bias condition observed in P3HT and other large band-gap organic semiconductor based photodiodes have been attributed to shallow and deep level trap states.[53]
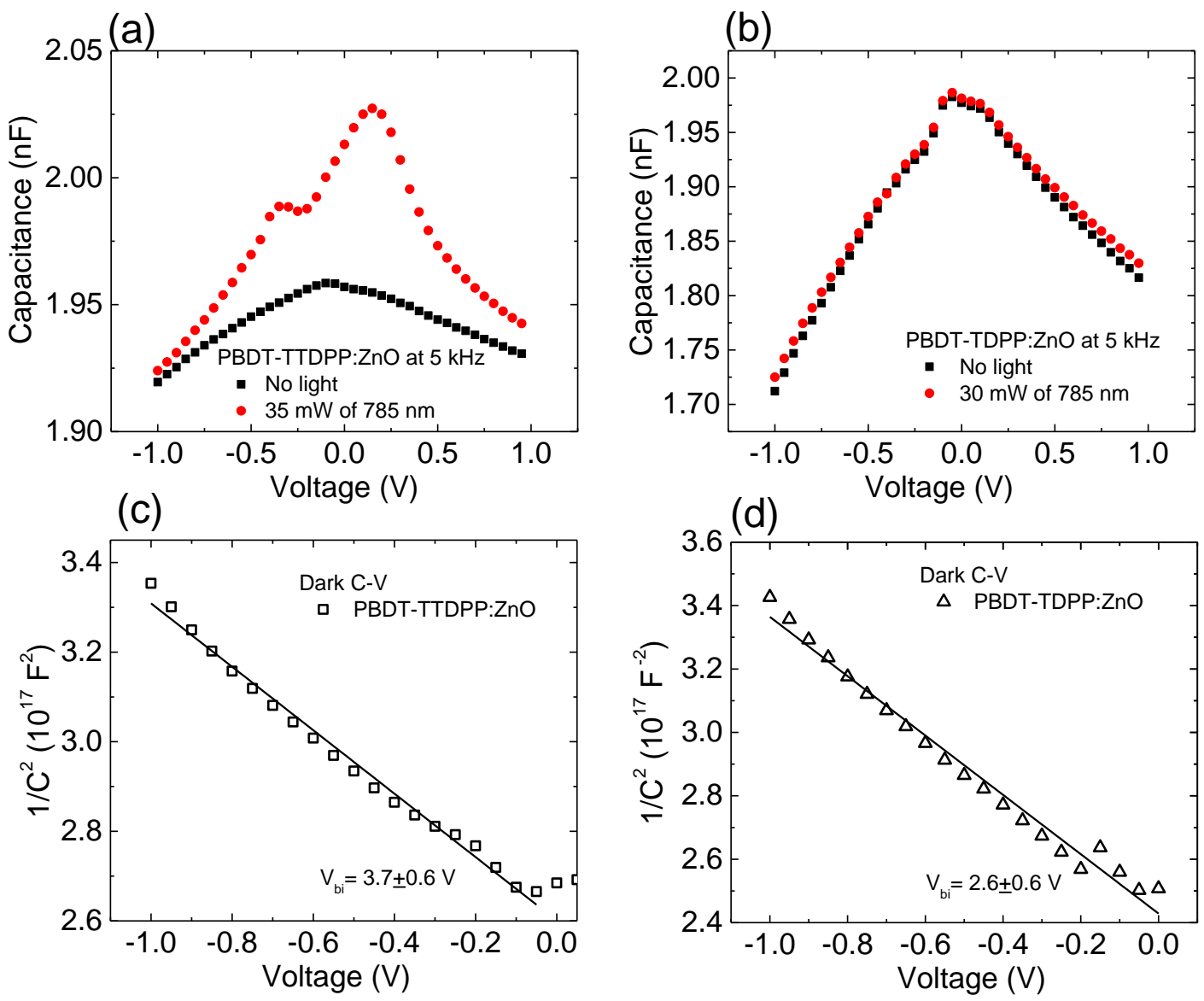

Figure 7. (a) and (b) Capacitance versus voltage for PTTDPP-TBDT:ZnO and PTDPP-TBDT diodes under dark and illumination conditions. (c) and (d) display the inverse of the square of the capacitance versus voltage.

In order to understand the differences in the CV measurements of the two DPP photodiodes, the Schottky diode parameters are obtained. Using eq. (1), the obtained built-in voltage is found to be slightly higher for PBDT-TTDPP:ZnO compared to PBDT-TDPP:ZnO (from Figure 7 (c) and (d)). These values are close to the ones obtained from a P3HT:ZnO photodiode, where $\mathrm{V}_{\mathrm{BI}}$ is determined as $2.9 \mathrm{~V}$. Thus, the lack of any photoresponse in the CV results for PBDT-TDPP:ZnO seems to be inherent to the copolymer, and consistent with the responsivity of the photodiode, which is at least two orders of magnitude smaller 
than PBDT-TTDPP:ZnO. Preliminary studies from UV-ozone treated $\mathrm{ZnO}$ photodiodes show reduced built-in voltages, pointing to the fact that the slightly high $\mathrm{V}_{\mathrm{BI}}$ in this work may be attributed to a lower conduction band-edge in $\mathrm{ZnO}$ due to oxygen vacancies. However, the differences in the performance of the photoresponsivities shown here do not originate from the diode properties but rather originate from their inherent charge transport mechanism.

The capacitance versus frequency behavior for the two DPP copolymer diodes is similar, at higher frequencies, as shown in Figure 8. A drop in the capacitance is observed around $200 \mathrm{kHz}$. The lack of saturation at low frequencies in PBDT-TDPP:ZnO is an indication of an incomplete trap response. Under light illumination, we again find no changes in the C-F curve for PBDT-TDPP:ZnO but a slightly higher capacitance at lower frequencies is observed for PBDT-TTDPP:ZnO, similar to light soaking C-F measurements in P3HT:PCBM.[54]

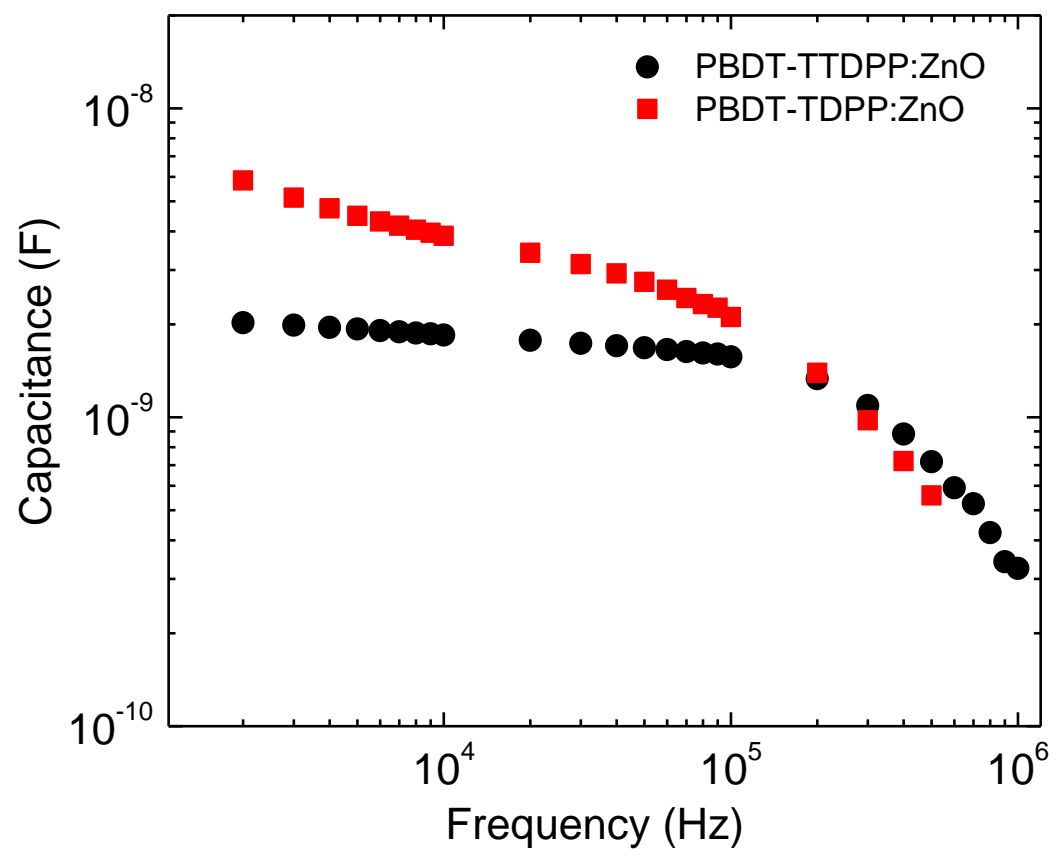

Figure 8. Capacitance versus frequency for PTDPP-TBDT:ZnO and PTTDPP-TBDT:ZnO diodes.

\subsection{TDDFT results}

The electronic and excitation energies of the DPP copolymers were computed using the Gaussian 09 package.[55] Dimeric units of PBDT-TDPP and PBDT-TTDPP were used to predict their electronic 
structure. Both systems were optimized using the B3LYP functional combined with the polarized 6$31 \mathrm{G}(\mathrm{d})$ basis set. We first calculated the ground state charge distribution of the dimers (with DFT) for both copolymers. Figure S5 shows the HOMO/LUMO and the next lower (higher) molecular orbitals obtained from ground state DFT calculations. The absorptivity of the two dimers was calculated using TDDFT as shown in Fig. 9. The calculations accurately predict lower optical band gap energy for PBDTTTDPP compared to PBDT-TDPP. Additionally, a higher absorptivity is observed for PBDT-TTDPP compared to PBDT-TDPP. Although the absorptivity of the dimers differs only by a factor of 1.6 , it shows the same trend as in experiments where the photoresponsivity in PBDT-TDPP is lower than in PBDT-TTDPP.

Since the molecular orbitals may not accurately reflect the many-body system due to the superposition of single-particle excitations, we computed the transition orbitals yielding a more complete picture in terms of the charge transfer character. The transition orbital pair was generated by subtracting the squares of the excited and the ground state molecular orbitals, which is similar to plotting the transition densities, as shown in Figure S6. In each case the hole is plotted in violet and the particle is plotted in turquoise. Since the charge transfer transition orbital pair corresponds to a fraction of the total excitation, we choose the isovalues accordingly for plotting the surface to reflect this fraction. Both dimers show a similar profile, with an electron deficient PBDT and a delocalized distribution of particlehole over the rest of the chain. The similarity in the transition densities thus suggests that more than the inra-charge transfer mechanism within the monomeric units it is the backbone planarity in PBDT-TTDPP, which results in a higher mobility and dissociation of excitons. This yields a higher photocurrent compared to the TDPP system. 


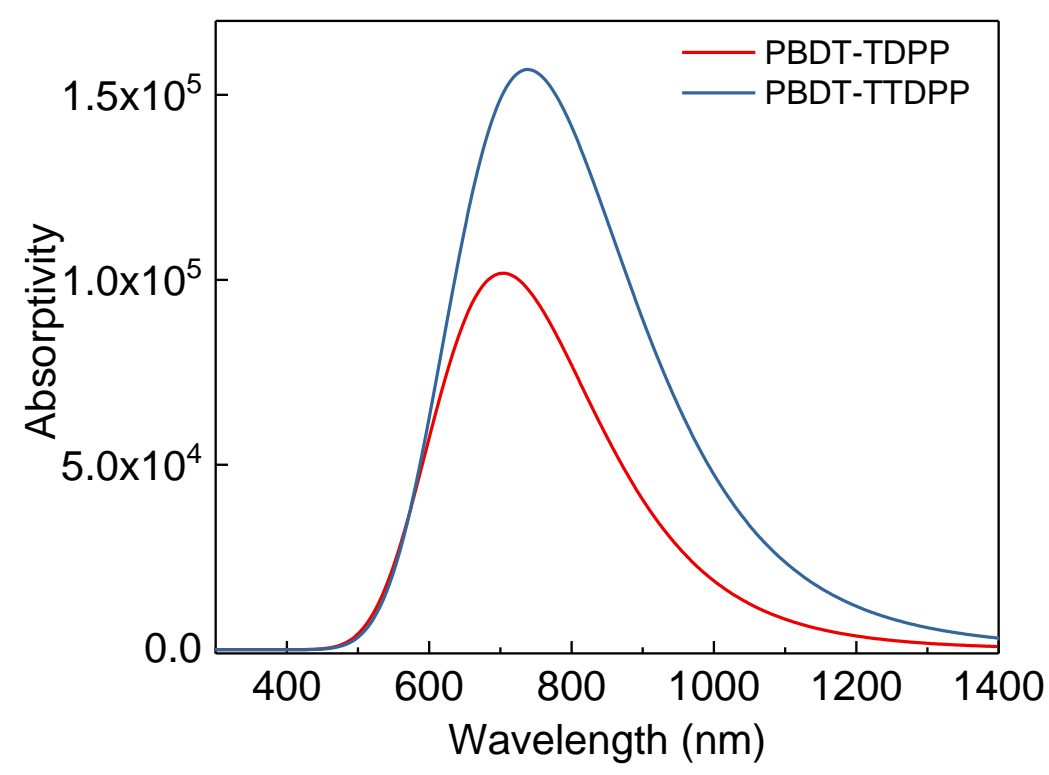

Figure 9. The calculated absorptivity of PBDT-TDPP and PBDT-TTDPP dimer using B3LYP/6$31 G(d)$.

\section{CONCLUSIONS}

Nanopatterned $\mathrm{ZnO}$ films provide realistic solutions for planar heterojunction, non-fullerene near IR and visible photodetectors. Solution processed nanopatterned $\mathrm{ZnO}$ films, varying between 300-500 nm, exhibit an improved interface to form a planar $p n$ heterojunction with $p$-type organic semiconductors. P3HT:ZnO photodiodes without the use of fullerenes yield an overall external quantum efficiency of 13\%. By comparing two near IR absorbing DPP based copolymers with varying backbone conformations in planar heterojunction photodiodes with an optimized nanopatterned $\mathrm{ZnO}$ interface, we show that the linkage unit between the DPP core and the BDT donor unit plays a role in charge dissociation. TDDFT calculations of dimer units show a higher absorptivity for PBDT-TTDPP compared to PBDT-TDPP. PBDT-TTDPP shows a peak responsivity around $730 \mathrm{~nm}$, which is at least 200 times larger than PBDTTDPP:ZnO photodiodes. The PBDT-TTDPP:ZnO photodiodes show a fast response in the near IR with rise and decay times around $100 \mathrm{~ms}$. 
Impedance measurements provide an alternate method for characterizing the photodiodes in terms of built-in voltages and trapping response of carriers at the $p n$ junction interface. Although both PBDTTTDPP and PBDT-TDPP photodiodes have similar built-in potential, their C-V and C-F characteristics differ. A lack of any photoresponse in the C-V measurements of PBDT-TDPP photodiodes further reflect that the transport of photocarriers in the copolymer is not efficient in a planar heterojunction architecture with $n$-type $\mathrm{ZnO}$.

Further improvement in DPP based near-IR photodiodes with nanopatterned $\mathrm{ZnO}$ may be achieved by solvent treatment and varying the annealing conditions of the copolymer layer to achieve an improved morphology as well as improving the $\mathrm{ZnO}$ layer by UV-ozone treatment. Adding a hole transport layer prior to the deposition of the Au electrode may further improve charge extraction. Although the two DPP copolymers have the same donor and acceptor units, the linker unit dictates the backbone conformation as well as charge dissociation and carrier mobility. DPP copolymers with a higher co-linearity of the monomer units may further help in enhanced absorption of light. Synthesis of DPP copolymers with deeper LUMO energy may facilitate electron injection in $\mathrm{ZnO}$ layer and improve their performance in planar heterojunctions for near IR photodetectors.

\section{ACKNOWLEDGMENT}

We acknowledge partial support of this work through the US National Science Foundation under Grant No. ECCS-1305642. S.P. thanks Department of Science and Technology for Swarnajayanti Fellowship. We thank Kartik Ghosh for providing the PLD grown $\mathrm{ZnO}$ films. 


\section{REFERENCES}

[1] L.J.A. Koster, O. Stenzel, S.D. Oosterhout, M.M. Wienk, V. Schmidt, R.A.J. Janssen, Morphology and Efficiency: The Case of Polymer/ZnO Solar Cells, Advanced Energy Materials, 3 (2013) 615-621.

[2] A. Guchhait, A.K. Rath, A.J. Pal, Near-IR activity of hybrid solar cells: Enhancement of efficiency by dissociating excitons generated in PbS nanoparticles, Appl. Phys. Lett., 96 (2010) 073505.

[3] J. Smith, A. Bashir, G. Adamopoulos, J.E. Anthony, D.D.C. Bradley, M. Heeney, I. McCulloch, T.D. Anthopoulos, Air-Stable Solution-Processed Hybrid Transistors with Hole and Electron Mobilities Exceeding 2 cm2 V-1 s-1, Adv. Mater., 22 (2010) 3598-3602.

[4] S.P. Singh, Z.-E. Ooi, S.N.L. Geok, G.K.L. Goh, A. Dodabalapur, Electrical characteristics of zinc oxide-organic semiconductor lateral heterostructure based hybrid field-effect bipolar transistors, Appl. Phys. Lett., 98 (2011) 073302.

[5] D. Kabra, L.P. Lu, M.H. Song, H.J. Snaith, R.H. Friend, Efficient Single-Layer Polymer Light-Emitting Diodes, Adv. Mater., 22 (2010) 3194-3198.

[6] H.J. Bolink, E. Coronado, D. Repetto, M. Sessolo, Air stable hybrid organic-inorganic light emitting diodes using ZnO as the cathode, Appl. Phys. Lett., 91 (2007) 223501.

[7] P. Kumar, S. Guha, F. Shahedipour-Sandvik, K.S. Narayan, Hybrid n-GaN and polymer interfaces: Model systems for tunable photodiodes, Org. Electron., 14 (2013) 2818-2825. [8] M. Nobuyuki, I. Yoshihiro, M. Takuya, K. Michio, S. Masatomo, Photovoltaic Action in Polyaniline/n-GaN Schottky Diodes, Appl. Phys. Express, 2 (2009) 092201.

[9] G. Heliotis, P.N. Stavrinou, D.D.C. Bradley, E. Gu, C. Griffin, C.W. Jeon, M.D. Dawson, Spectral conversion of InGaN ultraviolet microarray light-emitting diodes using fluorene-based red-, green-, blue-, and white-light-emitting polymer overlayer films, Appl. Phys. Lett., 87 (2005) 103505.

[10] I.O. Huyal, U. Koldemir, T. Ozel, H.V. Demir, D. Tuncel, On the origin of high quality white light emission from a hybrid organic/inorganic light emitting diode using azide functionalized polyfluorene, J. Mater. Chem., 18 (2008) 3568-3574.

[11] C. Zhang, A.J. Heeger, Gallium nitride/conjugated polymer hybrid light emitting diodes: Performance and lifetime, J. Appl. Phys., 84 (1998) 1579-1582.

[12] N. Sekine, C.-H. Chou, W.L. Kwan, Y. Yang, ZnO nano-ridge structure and its application in inverted polymer solar cell, Org. Electron., 10 (2009) 1473-1477.

[13] D.C. Lim, K.-D. Kim, S.-Y. Park, E.M. Hong, H.O. Seo, J.H. Lim, K.H. Lee, Y. Jeong, C.

Song, E. Lee, Y.D. Kim, S. Cho, Towards fabrication of high-performing organic photovoltaics: new donor-polymer, atomic layer deposited thin buffer layer and plasmonic effects, Energy \& Environmental Science, 5 (2012) 9803-9807.

[14] R. Shivanna, S. Rajaram, K.S. Narayan, Interface engineering for efficient fullerene-free organic solar cells, Appl. Phys. Lett., 106 (2015) 123301.

[15] S. Cho, K.-D. Kim, J. Heo, J.Y. Lee, G. Cha, B.Y. Seo, Y.D. Kim, Y.S. Kim, S.-y. Choi, D.C. Lim, Role of additional PCBM layer between $\mathrm{ZnO}$ and photoactive layers in inverted bulkheterojunction solar cells, Sci. Rep., 4 (2014) 4306.

[16] K.-D. Kim, D.C. Lim, J. Hu, J.-D. Kwon, M.-G. Jeong, H.O. Seo, J.Y. Lee, K.-Y. Jang, J.H. Lim, K.H. Lee, Y. Jeong, Y.D. Kim, S. Cho, Surface Modification of a ZnO ElectronCollecting Layer Using Atomic Layer Deposition to Fabricate High-Performing Inverted Organic Photovoltaics, ACS Appl. Mater. Interfaces, 5 (2013) 8718-8723. 
[17] O. Game, U. Singh, T. Kumari, A. Banpurkar, S. Ogale, ZnO(N)-Spiro-MeOTAD hybrid photodiode: an efficient self-powered fast-response UV (visible) photosensor, Nanoscale, 6 (2014) 503-513.

[18] C. Xu, P. Shin, L. Cao, D. Gao, Preferential Growth of Long ZnO Nanowire Array and Its Application in Dye-Sensitized Solar Cells, J. Phys. Chem. C, 114 (2010) 125-129.

[19] A. Yengantiwar, R. Sharma, O. Game, A. Banpurkar, Growth of aligned ZnO nanorods array on ITO for dye sensitized solar cell, Current Applied Physics, 11 (2011) S113-S116. [20] C. Kanimozhi, N. Yaacobi-Gross, K.W. Chou, A. Amassian, T.D. Anthopoulos, S. Patil, Diketopyrrolopyrrole-Diketopyrrolopyrrole-Based Conjugated Copolymer for High-Mobility Organic Field-Effect Transistors, J. Am. Chem. Soc., 134 (2012) 16532-16535.

[21] J. Li, Y. Zhao, H.S. Tan, Y. Guo, C.-A. Di, G. Yu, Y. Liu, M. Lin, S.H. Lim, Y. Zhou, H. $\mathrm{Su}, \mathrm{B} . \mathrm{S}$. Ong, A stable solution-processed polymer semiconductor with record high-mobility for printed transistors, Sci. Rep., 2 (2012) 754.

[22] S.P. Senanayak, A.Z. Ashar, C. Kanimozhi, S. Patil, K.S. Narayan, Room-temperature bandlike transport and Hall effect in a high-mobility ambipolar polymer, Phys. Rev. B, 91 (2015) 115302.

[23] P. Sonar, S.P. Singh, Y. Li, M.S. Soh, A. Dodabalapur, A Low-Bandgap

Diketopyrrolopyrrole-Benzothiadiazole-Based Copolymer for High-Mobility Ambipolar Organic Thin-Film Transistors, Adv. Mater., 22 (2010) 5409-5413.

[24] Y. Zhang, C. Kim, J. Lin, T.-Q. Nguyen, Solution-Processed Ambipolar Field-Effect Transistor Based on Diketopyrrolopyrrole Functionalized with Benzothiadiazole, Adv. Funct. Mater., 22 (2012) 97-105.

[25] D. Adil, C. Kanimozhi, N. Ukah, K. Paudel, S. Patil, S. Guha, Electrical and Optical Properties of Diketopyrrolopyrrole-Based Copolymer Interfaces in Thin Film Devices, ACS Appl. Mater. Interfaces, 3 (2011) 1463-1471.

[26] C. Kanimozhi, N. Yaacobi-Gross, E.K. Burnett, A.L. Briseno, T.D. Anthopoulos, U. Salzner, S. Patil, Use of side-chain for rational design of n-type diketopyrrolopyrrole-based conjugated polymers: what did we find out?, Physical Chemistry Chemical Physics, 16 (2014) 17253-17265.

[27] C. Kanimozhi, P. Balraju, G.D. Sharma, S. Patil, Synthesis of Diketopyrrolopyrrole Containing Copolymers: A Study of Their Optical and Photovoltaic Properties, J. Phys. Chem. B, 114 (2010) 3095-3103.

[28] J. Liu, Y. Sun, P. Moonsin, M. Kuik, C.M. Proctor, J. Lin, B.B. Hsu, V. Promarak, A.J. Heeger, T.-Q. Nguyen, Tri-Diketopyrrolopyrrole Molecular Donor Materials for HighPerformance Solution-Processed Bulk Heterojunction Solar Cells, Adv. Mater., 25 (2013) 58985903.

[29] B. Chen, Y. Yang, P. Cheng, X. Chen, X. Zhan, J. Qin, Designing a thiophene-fused DPP unit to build an A-D-A molecule for solution-processed solar cells, Journal of Materials Chemistry A, 3 (2015) 6894-6900.

[30] D. Moghe, P. Yu, C. Kanimozhi, S. Patil, S. Guha, Charge transfer complex states in diketopyrrolopyrrole polymers and fullerene blends: Implications for organic solar cell efficiency, Appl. Phys. Lett., 99 (2011) 233307.

[31] D. Moghe, G.K. Dutta, S. Patil, S. Guha, Photocurrent spectroscopic studies of diketopyrrolopyrrole-based statistical copolymers, Physical Chemistry Chemical Physics, 16 (2014) 4291-4298. 
[32] Y. Li, D. Moghe, S. Patil, S. Guha, C.A. Ullrich, Visualisation of charge-transfer excitations in donor-acceptor molecules using the particle-hole map: a case study, Mol. Phys., 114 (2016) 1365-1373.

[33] W.L. Leong, S.R. Cowan, A.J. Heeger, Differential Resistance Analysis of Charge Carrier Losses in Organic Bulk Heterojunction Solar Cells: Observing the Transition from Bimolecular to Trap-Assisted Recombination and Quantifying the Order of Recombination, Advanced Energy Materials, 1 (2011) 517-522.

[34] C. Melzer, E.J. Koop, V.D. Mihailetchi, P.W.M. Blom, Hole Transport in Poly(phenylene vinylene)/Methanofullerene Bulk-Heterojunction Solar Cells, Adv. Funct. Mater., 14 (2004) 865870.

[35] C. Lungenschmied, E. Ehrenfreund, N.S. Sariciftci, Negative capacitance and its photoinhibition in organic bulk heterojunction devices, Org. Electron., 10 (2009) 115-118.

[36] P.P. Boix, G. Garcia-Belmonte, U. Muñecas, M. Neophytou, C. Waldauf, R. Pacios, Determination of gap defect states in organic bulk heterojunction solar cells from capacitance measurements, Appl. Phys. Lett., 95 (2009) 233302.

[37] J. Yu, B. Zhao, X. Nie, B. Zhou, Y. Li, J. Hai, E. Zhu, L. Bian, H. Wu, W. Tang, Correlation of structure and photovoltaic performance of benzo[1,2-b:4,5-b[prime or minute]]dithiophene copolymers alternating with different acceptors, New Journal of Chemistry, 39 (2015) 2248-2255.

[38] R.K. Gupta, F. Yakuphanoglu, K. Ghosh, P.K. Kahol, Fabrication and characterization of p$\mathrm{n}$ junctions based on $\mathrm{ZnO}$ and $\mathrm{CuPc}$, Microelectronic Engineering, 88 (2011) 3067-3069.

[39] K.P. Musselman, S. Albert-Seifried, R.L.Z. Hoye, A. Sadhanala, D. Muñoz-Rojas, J.L. MacManus-Driscoll, R.H. Friend, Improved Exciton Dissociation at Semiconducting Polymer:ZnO Donor:Acceptor Interfaces via Nitrogen Doping of ZnO, Adv. Funct. Mater., 24 (2014) 3562-3570.

[40] G.K. Dutta, S. Guha, S. Patil, Synthesis of liquid crystalline benzothiazole based derivatives: A study of their optical and electrical properties, Org. Electron., 11 (2010) 1-9. [41] P.M. Beaujuge, C.M. Amb, J.R. Reynolds, Spectral Engineering in $\pi$-Conjugated Polymers with Intramolecular Donor-Acceptor Interactions, Acc. Chem. Res., 43 (2010) 1396-1407. [42] D. Li, W. Qin, S. Zhang, D. Liu, Z. Yu, J. Mao, L. Wu, L. Yang, S. Yin, Effect of UVozone process on the $\mathrm{ZnO}$ interlayer in[space] the inverted organic solar cells, RSC Advances, 7 (2017) 6040-6045.

[43] K.H. Hendriks, W. Li, M.M. Wienk, R.A.J. Janssen, Small-Bandgap Semiconducting Polymers with High Near-Infrared Photoresponse, J. Am. Chem. Soc., 136 (2014) 12130-12136. [44] Y. Li, C.A. Ullrich, The Particle-Hole Map: A Computational Tool To Visualize Electronic Excitations, J. Chem. Theory Comput., 11 (2015) 5838-5852.

[45] M.S. Vezie, S. Few, I. Meager, G. Pieridou, B. Dorling, R.S. Ashraf, A.R. Goni, H.

Bronstein, I. McCulloch, S.C. Hayes, M. Campoy-Quiles, J. Nelson, Exploring the origin of high optical absorption in conjugated polymers, Nat. Mater., 15 (2016) 746-753.

[46] S.K. Lai, L. Tang, Y.Y. Hui, C.M. Luk, S.P. Lau, A deep ultraviolet to near-infrared photoresponse from glucose-derived graphene oxide, J. Mater. Chem. C, 2 (2014) 6971-6977.

[47] R.F. Pierret, Semiconductor Device Fundamentals, Addison-Wesley1996.

[48] C. Zhao, X. Qiao, B. Chen, B. Hu, Thermal annealing effect on internal electrical polarization in organic solar cells, Org. Electron., 14 (2013) 2192-2197. 
[49] H. Zang, Y.-C. Hsiao, B. Hu, Surface-charge accumulation effects on open-circuit voltage in organic solar cells based on photoinduced impedance analysis, Physical Chemistry Chemical Physics, 16 (2014) 4971-4976.

[50] M.J. Lee, S. Seo, D.C. Kim, S.E. Ahn, D.H. Seo, I.K. Yoo, I.G. Baek, D.S. Kim, I.S. Byun, S.H. Kim, I.R. Hwang, J.S. Kim, S.H. Jeon, B.H. Park, A Low-Temperature-Grown Oxide Diode as a New Switch Element for High-Density, Nonvolatile Memories, Adv. Mater., 19 (2007) 7376.

[51] T. Muntasir, S. Chaudhary, Understanding defect distributions in polythiophenes via comparison of regioregular and regiorandom species, J. Appl. Phys., 118 (2015) 205504. [52] M. Devynck, B. Rostirolla, C.P. Watson, D.M. Taylor, Photo-response of a P3HT:PCBM blend in metal-insulator-semiconductor capacitors, Appl. Phys. Lett., 105 (2014) 183301. [53] B. Ray, A.G. Baradwaj, B.W. Boudouris, M.A. Alam, Defect Characterization in Organic Semiconductors by Forward Bias Capacitance-Voltage (FB-CV) Analysis, J. Phys. Chem. C, 118 (2014) 17461-17466.

[54] R.A. Street, Y. Yang, B.C. Thompson, I. McCulloch, Capacitance Spectroscopy of Light Induced Trap States in Organic Solar Cells, J. Phys. Chem. C, 120 (2016) 22169-22178. [55] GAUSSIAN09, GAUSSIAN 09, Revision B.01, Gaussian, Inc., Wallingford, CT, (2009) Revision B.01, Gaussian, Inc., Wallingford, CT. 
Table of Contents Graphic and Synopsis

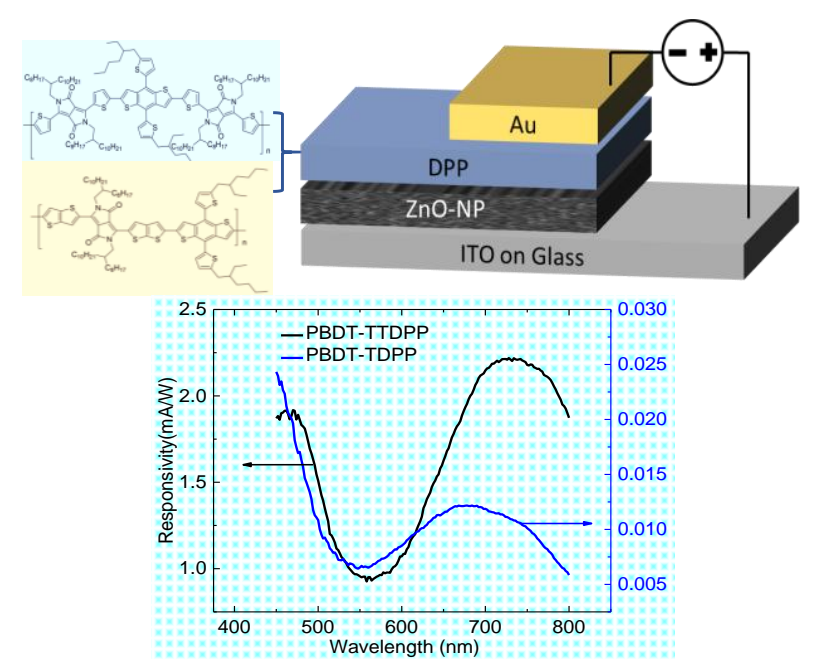

Nanopatterned $\mathrm{ZnO}$ and diketopyrrolopyrrole (DPP) donor-acceptor copolymer based planar heterojunctions provide a viable route for near-infrared photodetector applications. The rigid backbone of the DPP copolymer with a thienothiophene unit enhances charge transport properties. 